\title{
Patellar sleeve fracture
}

\author{
Priya I. Nath • Grant E. Lattin Jr.
}

Received: 18 March 2010 /Revised: 20 April 2010 /Accepted: 14 May 2010 /Published online: 5 June 2010

(C) US Goverment 2010

A 14-year-old boy was evaluated for knee pain that occurred while running. On examination, tenderness to palpation was noted along the patella and extensor mechanism. The lateral radiograph demonstrates prepatellar soft-tissue swelling with separation of the anterior and inferior calcified fibrocartilage of the patella (Fig. 1, lines).

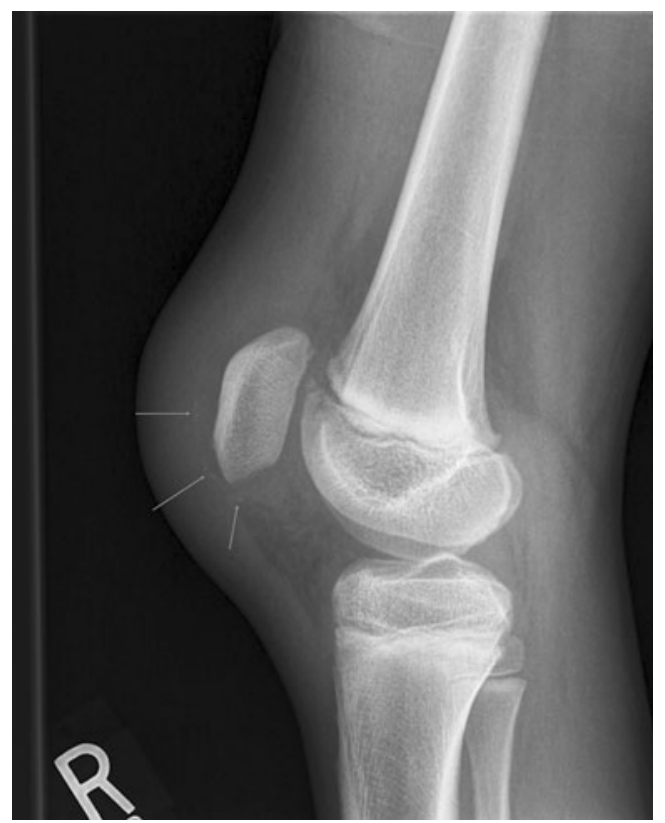

Fig. 1 Lateral radiograph

P. I. Nath $(\bowtie)$

United States Air Force,

Uniformed Services of the Health Sciences, School of Medicine,

4301 Jones Bridge Road,

Bethesda, MD 20814, USA

e-mail: priya.nath@usuhs.mil

\section{G. E. Lattin Jr.}

United States Air Force,

Department of Radiology and Radiological Sciences, Uniformed Services University of the Health Sciences, Bethesda, MD, USA

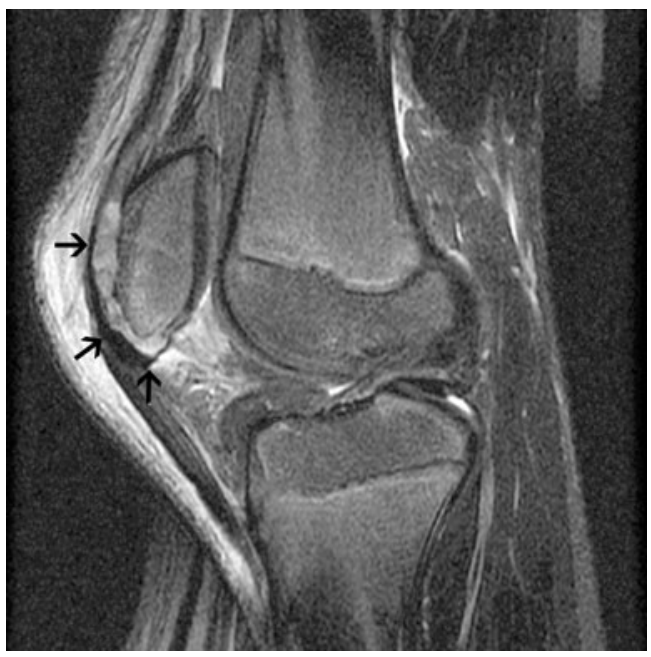

Fig. 2 Sagittal T2-W MRI

MRI confirms a patellar sleeve fracture with laminar tearing of the extensor mechanism (Fig. 2, arrows).

A patellar sleeve fracture is an uncommon cartilaginous avulsion resulting from hyperextension of the knee. Often identified as a distal pole avulsion, these represent a disengagement of the bone-forming physeal cartilage and associated zone of provisional calcification in addition to the overlying epiphyseal cartilage [1]. Radiographs might underestimate the degree of injury. MRI shows the full extent of soft-tissue and cartilaginous injury. Management can be conservative or surgical depending on injury severity [2].

\section{References}

1. Dwek JR (2010) The periosteum: what is it, where is it, and what mimics it in its absence? Skeletal Radiol 39:319-323

2. Bates DG, Hresko MT, Jaramillo D (1994) Patellar sleeve fracture: demonstration with MR imaging. Radiology 193:825-827 